\title{
El conexionismo
}

\author{
José Luis Fernández Trespalacios
}

\section{Introducción}

¿Por qué dentro de un conjunto de trabajos que tratan sobre el pasado, el presente y el futuro de la psicología, parece conveniente incluir el tema del conexionismo? En primer lugar, al hablar del presente y futuro de la psicología hay que hablar necesariamente del conexionismo, porque si no es la última novedad en psicología, sí al menos es una de las últimas novedades.

Sin duda en la psicología aplicada hay temas de la última actualidad que no tienen nada que ver con el conexionismo. Así son muchos de los últimos temas que ha abordado la psicología aplicada, como son, por ejemplo, la medicina conductual, la psicología de la salud o la psicología de las organizaciones. Basta pasearse por una de las importantes universidades mundiales, para comprobar la actualidad que hoy tienen en psicología los temas aplicados y específicos.

Pero si en la psicología aplicada, al preguntarnos por la última novedad en psicología, podriamos hablar de muchas cosas que nada tienen que ver con el conexionismo, en la psicología básica, por el contrario la última novedad lo constituye un solo tema y este tema es el conexionismo. $\mathrm{Si}$, pues, en un conjunto de trabajos se pretende tratar el presente más actual de la psicología básica parece obligado tratar del conexionismo.

Existe, sin embargo, para mí particularmente otra razón que me obliga a enfrentarme con este tema. Si se ha criticado, durante mucho tiempo, las debilidades que presentaba el paradigma de la psicología cognitiva de los últimos años, parece obligado adoptar, también, una posición positiva y tratar de ofrecer esta nueva alternativa que en el estudio de la mente y conducta humana representa hoy el conexionismo. Si hemos estado al lado de los que durante los años sesenta y setenta han hecho caer muchas ilusiones de las albergadas por la psicología cognitiva, parece obligado apresurarse en mostrar las posibilidades de esperanza e ilusión que muchos encuentran en la psicología conexionista.

La psicología cognitiva de los últimos años nos ha llevado, como señala entre muchos el psicólogo italiano R. Luccio (1982), a intentar entender la mente humana con "modelos cada vez más abstractos y alejados de la realidad" (p. 255). Nada de extraño que la psicología cognitiva se haya ido convirtiendo poco a poco o bien en un lenguaje casi filosófico, de tipo racionalista, como se muestra en las obras de Fodor o Pylyshyn, o bien en un estudio de programas de ordenador, como ocurre hoy bajo el férreo dominio de la Inteligencia Artificial. No deja de ser curioso, en este sentido, que un cierto número de psicólogos actuales estén ahora estudiando el aprendizaje en modelos de ordenador más bien que examinando las tareas y los procesos explícitamente en los seres humanos.

Frente a todo ello, el conexionismo pretende alejarse del modelo del computador 
convencional y de lenguajes racionalista como el de la lingüistica chomskiana, para acercarse al modelo del cerebro animal y humano y a lenguajes como el de la neurofisiología. El impacto ha sido tan profundo que sería fácil imaginar que un Instituto de investigación psicológica tendría hoy que mirar al futuro con dos tipos de departamentos: un departamento de psicología conexionista que estudiaría la dimensión psicológica, pero con un ojo puesto en la fisiología del cerebro y un departamento de neurociencia que estudiaría la base fisiológica de la mente y conducta humana, pero con las miras puestas en la psicología.

Como conclusión, pues, de esta larga introducción, el sentido que tiene el presente trabajo es doble: primero, intentar exponer, aunque sea muy someramente, el último avance de la psicología básica; segundo, ofrecer la esperanza que estos últimos avances en psicología representan para el objetivo de nuestra ciencia. ¿Qué ocurre dentro de la mente humana? ¿Cómo describir y explicar lo que es más interesante del ser humano: es decir, su habla, razonamiento y pensamiento?

\section{Un poco de historia}

Podemos afirmar que las dos grandes guerras europeas que hemos sufrido en cl presente siglo han tenido repercusiones importantísimas para la psicología. La primera trajo consigo un gran desarrollo de los llamados tests mentales. La segunda guerra mundial ha traido, entre otras cosas, el interés de los psicólogos en la Ingenicría Humana. La guerra trajo consigo el uso de muchas nuevas máquinas y era necesario encontrar las máquinas a las que más fácilmente se adaptara el ser humano, para encontrar un rendimiento mejor.

En Norteamérica J. J. Gibson tuvo que dedicarse al estudio de la percepción de los pilotos de aviones de guerra en el aterrizaje y en el despegue. Tal tipo de estudios le llevó a afianzarse en su concepción ecológica en el estudio de la percepción. En Inglaterra el uso de las máquinas llevó a un grupo de psicólogos de Cambridge a preocuparse de temas como la atención dividida, tareas de seguimiento de una señal etc.

Estos trabajos llevaron a hacer pensar que el hombre posee un mecanismo de decisión que emplea al menos medio segundo en elaborar la información que le llega. Además había que admitir que tal mecanismo de decisión actua de una manera discreta. Dicho con otras palabras, el mecanismo de decisión elabora una información y luego otra, pero hasta que no ha elaborado la primera, no puede elaborar la segunda.

Las observaciones que llevaron a semejantes supuestos eran muy simples. Cuando un individuo realizaba la tarea de acoplar una señal con un objetivo que se mueve, tenía que realizar frecuentes correcciones. Pues bien, sólo podía realizar una corrección cada medio segundo. Las consecuencias fueron importantísimas. El hombre iba a ser como un procesador de información. Una nueva psicología comienza a bullir. Los psicólogos de la percepción quieren medir la cantidad de información proporcionada por el estímulo y, en virtud de ello, poder explicar alguno de los principios de organización perceptual. Se pone de moda de nuevo la medida de tiempos de reacción con la finalidad de medir el tiempo que consume cada proceso cognitivo. Se adopta un nuevo lenguaje; el de los diagramas de flujo para poder exponer teorías sobre la atención y la memoria. 
Finalmente en 1960 la obra de Miller, Galanter y Pribam sobre los planes y estructura de la conducta establece la analogía entre el hombre y cl ordenador y se daba entrada de alguna manera al lenguaje, mas o menos de tipo racionalista, de la gramática generativo-transformacional. Con esto se cerraban los años cincuenta y con ellos la época fascinante e ilusionada en que se abre camino una nueva psicología: la psicología cognitiva. El modelo conductista del estímulo y la respuesta no valía para explicar toda la compleja conducta del hombre. Era necesario conoccr qué ocurre dentro de la mente humana. El hombre como procesador de información era el nuevo concepto, el nuevo método la analogía del ordenador.

Pero a partir de' aquí las cosas cambían. Durante los años sesenta el conductismo retrocede y se implanta el nuevo paradigma de la psicología cognitiva. Esto es verdad, pcro ello no se corresponde con la realización de los avances que se esperaban. Pronto en la primera mitad de los años sesenta aparecen las primeras críticas y las primeras desilusiones. Las cosas no van. Los modelos de cajas, formalismo tomado de la metáfora del ordenador, no funcionan del modo necesario para explicar lo que ocurre en la mente humana. No es real hablar de unas cajas o macroestructuras que realiza cada una de ellas una función, como memoria a corto plazo o atenuador o memoria a largo plazo etc. Se intenta dejar de hablar de estructuras y se pasa a hablar de niveles de procesamiento, pero las cosas tampoco se arreglan.

Poco a poco los modelos de la psicología cognitiva se hacen más abstractos, más fragmentarios y, sobre todo, más alejados de la realidad. Es como si la psicología cognitiva hubiera comenzado una huída hacia adelante que le lleva unas veces a que el formalismo informático se vaya, poco a poco, convirtiendo en un lenguaje casi filosófico y, otras veces, le lleva a someterse a la Inteligencia Artificial centrándose más en el estudio de programas de ordenador que en la mente humana.

Nada de cstraño que al comienzo de los años ochenta, un historiador de la psicología como R. Luccio (1982) llegue a afirmar textualmente: "creo no equivocarme si considero más que probable que el cognitivismo, tal como lo hemos conocido, ha agotado o está a punto de agotar su función" (p. 255).

Frente a esa desilusión por la psicología cognitiva, por lo menos tal como se ha venido haciendo hasta ahora, aparece en los años ochenta una nueva ilusión: el conexionismo. Para algunos es un verdadero cambio de paradigma en la psicología, otros no se atreven a afirmar tanto. Podemos decir que el conexionismo comienza verdaderamente a propagarse a partir del verano de 1986. Estamos en 1987 es todavía muy pronto para saber todo lo que representa y todo lo que va a ser capaz de dar de sí. Hoy por hoy el hecho verdadero es que una nueva esperanza se ha abierto a la ciencia de la psicología.

La historia del conexionismo es muy pequeña. Ha tenido ciertos antecedentes dentro de la psicología en las obras de Rosenblatt de los años cincuenta y comienzo de los sesenta. Puede decirse que el primer paso serio se da con la obra de Hinton y J. A. Anderson de 1981 sobre modelos paralelos y memoria asociativa. Sin embargo, la concepción conexionista todavía presentaba serias dificultades que no se superarían hasta 1985. Por fin en 1986 se dispone ya de suficiente información sobre el conexionismo y comienza la propagación de su modo de construir psicología. Dejemos, pues, la historia y pasemos ya a describir, aunque necesariamente de modo somero, lo que es el co- 
nexionismo.

\section{El conexionismo: su descripción}

No obstante el avance de la tecnología actual, el hombre es mejor que las máquinas. Sin duda hay muchas razones que pueden dar cuenta de nuestra afirmación anterior. Pero hay una razón fundamental y ésta es que el cerebro emplea una arquitectura de computación que está mucho más adaptada a lo que el hombre hace, que las arquitecturas de computación que emplean las máquinas.

En este sentido Feldman y Ballard (1982) señalan que los modelos comutacionales de la psicología deben ser plausibles por lo menos en los recursos que requicren. Pues bien, uno de los recursos críticos en la computación es el tiempo necesitado para realizarla y el hombre, con un cerebro lento en comparación a la velocidad de muchos ordenadores, no puede gastar mucho tiempo en la realización de sus conductas.

Muchas de las conductas complejas que el hombre realiza puede ejecutarlas en unos pocos cientos de milisegundos. Esto significa que el hombre realiza las conductas complejas en menos de cien pasos de tiempo, si hablamos en términos de computador. Sin embargo, los programas de simulación y los modelos de Inteligencia Artificical necesitarian millones de pasos de tiempo para realizar esas mismas conductas complejas. Nuestro cerebro, aunque es más lento, requiere menos tiempo que un programa de un ordenador para realizar muchas de las conductas complejas ¿Cuál es la razón de ello?

La premisa fundamental del conexionismo es que su modelo consta de un número masivo de unidades elementales que son como neuronas y que esas unidades no transmiten grandes cantidades de información simbólica, sino que realizan la computación conectándose entre ellas de un modo apropiado. Dicho con otras palabras, un sistema de computación lento requiere poco tiempo en comparación del computador convencional, si dicho sistema consta de números masivos de unidades y conexiones en lugar de unas pocas macroestructuras que almacenan grandes cantidades de información simbólica y sobre las que opera un centro director del proceso que contiene un conjunto de reglas explícitas. Pero vayamos por pasos.

\subsection{Necesidad de las interacciones}

Una cantidad mínima de información, lo que se llama un bit de información, necesaria para resolver un simple problema con dos alternativa es una restricción de probabilidad. Por ejemplo, si queremos averiguar en cual de los dos semicírculos en que el diámetro divide un círculo hay un punto o señal, basta un bit de información para restringir la probabilidad de esta alternativa. Un sólo bit de información nos proporciona el que podamos resolver el problema de saber en cuál de los dos semicírculos está el punto. Dicho con otras palabras, un bit de información constituye una restricción de la probabilidad. Con el bit de información el punto pierde la probabilidad de estar en el otro semicírculo. Naturalmente para resolver problemas más complejos en que las alternativas son muchas hacen falta múltiples restricciones. Ahora bien, en la teoría de la información esas restricciones actuan binariamente y, por tanto, tienen que actuar secuencialmente. En el ejemplo anterior tendriamos que ir informando si hiciéramos el problema más complejo; el punto está en el círculo no en las otras figuras 
que no son círculos; dentro del círculo está en cl semicírculo derecho, no en el izquierdo; dentro del semicírculo izquierdo está en cl cuadrante superior no en el inferior etc. Es decir, la solución de un problema complejo requiere múltiples restricciones, pero un sistema de información convencional lo hace secuencialmente.

Sin embargo, hay restricciones múltiples simultáneas, como señalan Rumclhart y McClelland (1986), para una conducta tan simple como extender la mano para apretar un botón. Son las restricciones que ponen la estructura del brazo y los músculos, la forma del botón, su posición etc. Dichos autores hacen hincapié en las múltiples restricciones simultáneas que son necesarias para el procesamiento del lenguaje. La sintaxis constriñe el significado. Las reglas de la sintáxis son necesarias para que entendamos. Dicho con otras palabras la sintaxis determina de alguna manera la semántica. Pero las relaciones semánticas determinan a su vez la estructura sintáctica. Los autores citados ponen un ejemplo claro: en las siguientes dos oraciones es la semántica la que determina la estructura sintáctica. Las dos oraciones son: "vi el gran cañón volando a Nueva York" y "vi a una oveja pastando en el campo".

La conclusión es que el conocimiento se consigue mediante la interacción de múltiples fuentes. Se requieren mecanismos en que un aspecto de la información pueda actuar sobre otros y al revés. Esto es lo que pretende el conexionismo: procesar la información a través de las interacciones de un número masivo de elementos simples que se envian entre ellas, mediante conexiones, señales excitatorias o inhibitorias. Dicho con otras palabras, gracias a la interacción de múltiples unidades elementales conectadas entre sí se pueden dar restricciones múltiples simultáneas. Por esto el modelo de procesamiento conexionista se llama procesamiento Paralelo Distribuído (en inglés PDP). Distribuído porque se expande sobre un gran número de unidades y paralelo porque estas no se conectan serial o secuencialemente sino simultáneamente y en diversos niveles.

\subsection{Relación con la neurofisiología}

Si los modelos de la psicología cognitiva, tal como ha sido hasta ahora, no eran plausibles fisiológicamente, los modelos conexionistas sí lo son y tienen una inspiración neural. Ahora no se trata de encontrar una máquina que nos sirva para explicarnos el cerebro, como han sido los formalismos computacionales, convencionales. Ahora se trata de lo contrario, se trata de hacer un formalismo computacional que imite al cerebro, que funcione como el cerebro.

Esta inspiración neurofisiológica del conexionismo ha constituído sus primeras raíces. A finales del siglo pasado y en la primera mitad del nuestro ya hubo autores como Jackson que descartó la teoría simplista de las localizaciones cerebrales y defendió concepciones distribuídas y multiniveles. En la segunda mitad del presene siglo, en 1966, el ruso Alejandro Luria ha defendido que un proceso cognitivo o conductual resulta de la coordinación de muchos componentes, localizados en muy diferentes regiones del cerebro. En 1949 Donald Hebb aportó conceptos que han tenido gran repercusión como la regla de modificación sináptica y los conceptos de asambleas celulares y sintonización de fases. En 1950 Lashley afirmaba que no existen células especiales para almacenar memorias especiales e insiste en una concepción de representación distribuída. 
Es verdad que estas concepciones quedaron en la neurofisiología y que fucron conceptualizaciones demasiado vagas, poco claras y demasiado especulativas a veces. Pero, sin embargo, no han faltado psicólogos que han llamado la atención en que el camino correcto era observar cómo funciona el cerebro y no cómo funciona una máquina. En 1962 Rosenblatt publica unos principios de ncurodinámica y nos abre a la esperanza de un enfoque neural en el estudio de la computación. Finalmente, aunque no faltaron algunos intentos en la década pasada, es a partir de los primeros años ochenta cuando se intensifica el estudio de la computación inspirada neuralmente. J. A. Anderson (1983) ha publicado un trabajo con el significativo nombre de "Computación psicológica y cognitiva con modelos neurales". Uno de los objetivos de este trabajo es, precisamente, discutir el hardware del sistema nervioso real y, luego, desarrollar modelos neuronales simples para procesos cognitivos que trabajen con las mismas restricciones con que lo hace la naturaleza.

Nada de extraño que W. Schneider (1987) nos diga que el conexionismo es el cambio más dramático que ha ocurrido en la psicología en los últimos veinte años. Porque si hasta ahora se ha intentado describir el cerebro en términos de una máquina, una red telefónica o, incluso, una red semántica, ahora es al revés, se trata de describir la computación según la interacción de unidades que actúan como las neuronas. Antes era intentar ver qué clase de máquina es el ccrebro, ahora se trata de intentar ver cómo funcionan los modelos que son como o imitan al cerebro.

Pero el conexionismo es psicología y estudia la computación que realizan los procesos cognitivos, no es neurofisiología. El conexionismo no estudia el potenial de acción de la diferencia en polarización de la membrana, ni que elementos químicos actúan como transmisores, neuromoduladores o neurohormonas. Tampoco estudia macroestructuras cerebrales como los componentes del sistema límbico ni conexiones ncuroanatómicas entre las diversas regiones del cortex. Se trata de construir un modelo de computación, cuyo hardware pudiera ser el sistema nervioso superior, pero no de estudiar ese sistema nervioso.

\subsection{Características básicas del conexionismo}

Hay varias características que son comunes a todos los modelos conexionistas y que, por tanto, pueden servir para definir el conexionismo. Algunas de ellas las hemos señalado ya, pero es conveniente hacerlo ahora aquí de una manera sistemática.

En primer lugar, el procesamiento ocurre de modo simultáneo en una población de elementos simples. W. Schneider pone como ejemplo el procesamiento de la letra $\mathrm{H}$ en una población de ocho elementos que pueden tener cada uno de ellos valores binarios. En este caso serían, simetría vertical, simetría horizontal, simetría diagonal, no redondez, no cerrada, sin inclinación. Rumelhart y McClelland (1986) ponen el ejemplo de una hipótesis sobre la identidad de una palabra que se distribuiría en la activación de un gran número de unidades.

En segundo lugar, la actividad consiste en que esas unidades elementales envien sus señales de activación e inhibición a cada otra unidad, hasta que la actividad combinada de una población o conjunto de unidades empuja a todo el sistema hacia una acción. Es, pues, necesario distinguir entre activación y acción. La activación es de cada una de las unidades del sistema, la acción es del sistema en conjunto, de todo el sistema. 
Una acción consiste en que el sistema se establece dentro de una particular distribución de activación a través de las unidades del sistema. Dicho con otras palabras, la acción del sistema resulta de un patrón de activación distribuído sobre las unidades del sistema.

Tales acciones no son el producto de un "centro de control del proceso" o especie de ejecutivo que manipula la información almacenada representativamente en grandes almacenes com memoria a largo plazo etc. Precisamente aquí hay una gran diferencia entre el conexionismo y la psicología cognitiva anterior. Aquí cada unidad determina su propia actividad sobre la base, por un lado de su propio estado de activación inicial $y$, por otro lado, del input que recibe de las otras unidades.

Esta concepción de la activación de las unidades y de la acción del sistema, que tiene un marcado carácter neurofisiológico, se pude interpretar psicológicamente de dos maneras. Una de ellas, consiste en considerar que cada unidad en el sistema representa una hipótesis y el grado de activación representa su confianza. Se trata de una interpretación cognitiva y es la que siguen Rumelhart y McClelland (1986). Si yo quiero escribir la palabra "malo" se activa una unidad para la palabra y ésta, a su vez, activa cuatro unidades para las letras y éstas, finalmente,, activan los movimientos de la mano y los dedos. Naturalmente hay un juego de mas fuerte activación para la letra y movimientos con los que se ejecuta su escritura en el momento correspondiente.

Quizá la interpretación cognitiva de la activación se entiende mejor con el ejemplo que pone Bechtel (1985) con el modelo de reconocimiento de las palabras. La conexión excitatoria indica que las hipótesis se sostienen unas a otras (en realidad lo que se sostiene es la activación de unas a otras, pero aquí se da esta interpretación cognitiva como si cada unidad fuese una hipótesis y su activación es su sostenimiento). En el reconocimiento de la palabra "por", si queremos poner un ejemplo, la hipótesis de la letra " $p$ " en la primera posición y la unidad que representa la palabra "por" se activan mutuamente y la hipótesis se sostiene.

La segunda manera de interpretar psicológicamente la actividad de las unidades no proporciona una interpretación cognitiva para cada una de las unidades individuales, sino sólo para los patrones de actividad que se expanden sobre un conjunto de unidades. A esta interpretación se le llama perspectiva de representación local. Pero, como vemos, es también un modo de hablar o interpretar, porque no se trata de ninguna representación cognitiva, que en todo caso sería algo emergente, sino de un patrón de activación.

La tercera característica del conexionismo consiste en que el conocimiento no se almacena de modo representativo simbólico en macroestructuras o almacenes, sino que se almacena en asociaciones o fuerzas de conexión entre esos elementos o neuronas. Dicho con otras palabras, todos los patrones de activación que el sistema ha adquirido para responder al estímulo se suman en una matriz de asociación; esto es, todo el conocimiento se almacena en un pequeño número de matrices de asociación. En realidad no hay nada almacenado a no ser fuerzas de conexión, esto es lo que determina que al producirse la activación se forme un patrón entre las unidades más fuertemente conexas.

La cuarta característica es que la combinación de los inputs que realizan las unidades es una combinación simple; esto es, una suma o multiplicación y las transformaciones 
de sus inputs que realizan dichas unidades no es lineal. Dicho con otras palabras no hay comparación de símbolos, sino suma o multiplicación de actividades solamente. Rumelhart y McClelland en la obra ya citada coinciden con W. Schneider (1987) en señalar como característico del conexionismo tanto el que el conocimento no se almacena en una copia estática de un patrón, ni hay patrones almacenados en grandes estructuras, como el que las combinaciones de la activación y sus transformaciones se hacen de la manera más simple.

Finalmente la quinta característica del conexionismo consiste en que el aprendizaje implica modificar las conexiones. Esto es, aprender es modificar las conexiones de tal manera que se facilite el que un patrón de inputs antiguo evoque un patrón de outputs nuevo. Aprender es establecer las correctas fuerzas de conexión, de tal manera que los patrones correctos de activación se produzcan bajo las circunstancias correctas.

En el aprendizaje no se trata de formular esquemas o reglas explícitas que se almacenan y que utilizaría el centro de control del proceso o ejecutivo cuando manipula las representaciones almacenadas, tal como se ha pensado en la psicología cognitiva anterior. No es que yo aprenda la regla de añadir la sílaba "ed" para formar el preterito de los verbos ingleses y hay un conjunto de reglas almacenadas para que el "ejecutivo" proceda, es simplemente que la activación de pretérito está asociada a la activación de la sílaba "ed" al final de la forma verbal. La regla puede ser algo emergente, lo real es la sociación de activaciones. Aprender es establecer la conexión correcta.

Como resumen de todas estas características del conexionismo podemos decir que éste se opone radicalmente al concepto de computador de von Neuman que tan radicalmente ha modelado a la psicología cognitiva, tal como se ha desarrollado hasta ahora. No hay una estructura de datos y un ejecutivo, como esencialmente proponía el computador de von Neuman. Por eso el aprendizaje no consiste en instruir al ejecutivo para que realice ciertas operaciones sobre los elementos de la estructura de datos.

En este sentido, dentro de la psicología cognitiva, Pylyshyn (1984) dice que lo que caracteriza la arquitectura de la mente son las reglas y las representaciones. Por esto la psicología cognitiva ha explicado los procesos mentales defendiendo que la mente posee un almacen de representaciones o símbolos y un conjunto de reglas que se pueden usar para operar según la lógica sobre esas representaciones. El conexionismo rechaza que la mente sea una máquina que emplee reglas explícitas sobre representaciones formales. Lo que en la mente hay son unidades elementales y conexiones entre ella. Por eso es importantísimo distinguir entre la descripción de la actividad misma y la descripción de lo que emerge de dicha actividad de la mente.

Finalmente y en relación con todo lo dicho hay un último punto en que el conexionismo se parta radicalmente de la psicología cognitiva. Es el tema del innatismo. El conexionismo es asociacionista $y$, por tanto, empirista. La psicología cognitiva es racionalista y, por tanto, tiene ciertas tendencias innatistas.

Como ya hemos dicho, para la psicología cognitiva la cognición es un proceso de la mente que opera sobre las representaciones. Ahora bien, la mente tiene un conjunto de procedimientos para procesar representaciones. Estos procedimientos se toman como algo innato a la mente. Esta concepción nativista es fácil siempre que se haga una distinción entre representaciones y procesos. Se considera a la mente como una máquina con ciertas capacidades fijas. Dadas las representaciones, la mente tiene ciertas 
capacidades para hacer con ellas una serie de cosas, como razonamientos, discursos, etc. Pensar que estas capacidades fijas en cuanto procedimientos de procesar son innatos es cosa fácil.

El conexionismo no distingue entre representaciones y procesos. El sistema, como hemos dicho repetidas veces, no tiene representaciones almacenadas sobre las que se realizan operaciones según ciertos procedimientos. El sistema tiene sólo fuerzas de conexión entre unidades que permiten la creación de representaciones o, más exactamente, patrones de activación. Las fuerzas de conexión hacen el trabajo de los procesos, pero también crean las representaciones. No hay distinción entre reglas o procedimientos constantes y representaciones variables.

Lo dicho ticne también importantes implicaciones para el aprendizaje porque en el conexionismo no puede haber mecanismos de aprendizaje con capacidades computacionales poderosas, que incluso podían poseer conocimiento innato. Para el conexionismo lo que hay es modulación de las fuerzas de conexión de tal manera que se produzca un nuevo ajuste de dichas fuerzas entre las unidades.

Este cambio de concepción ya se había producido en la bioquímica del cerebro. Se pensó que la memoria y el aprendizaje tenían su hardware en los ácidos nucléicos. Así era fácil de explicar a nivel bioquímico el almacenaje de representaciones estables e incluso se podía pensar en conocimiento innato. Tal concepción bioquímica de la memoria y el aprendizaje llegó a estar tan arraigada que se llegó a vender en las farmacias medicamentos como la "nucleserina" para aumentar la capacidad de memoria y aprendizaje.

Sin embargo, ya hace tiempo que la bioquímica ha señalado que en la concepción bioquímica de la memoria y el aprendizaje lo importante no es el núcleo, sino la membrana de la neurona. Lo importante no está en los ácidos nucléicos que se tienen o adquiere. Lo importante está en los mecanismos de conexión del axon de una neurona con las dendritas de la neurona con la que se conecta. De patrones estáticos la bioquímica del cerebro ha pasado a patrones de fuerzas de conexión; esto es, a patrones dinámicos.

La revolución que hizo en su día la bioquímica del cerebro intenta hacerla ahora el conexionismo en el campo de la psicología en relación a los modelos computacionales de la psicología cognitiva. La explicación de la percepción, la memoria, el aprendizaje etc. ya no se hace a partir de procedimientos o reglas estáticas, almacenadas e innatas. Ahora todo ello se explica en virtud de las fuerzas de conexión entre las unidades elementales.

\subsection{Un ejemplo de un modelo conexionista}

Hoy hay ya modelos muy diversos dentro del campo conexionista. Hay modelos de procesamiento distribuído paralelo sobre percepción, memoria, lenguaje etc. Quizá uno de los más simples y fáciles de entender sea un modelo de control motor. Se trata de describir cómo la mente controla la conducta de una persona que escribe a máquina. Aquí vamos a hacer sólo una exposición resumida y cualitativa. Pero este modelo, no obstante ser muy sencillo, tiene la ventaja de ser una buena ilustración del hecho de que una conducta serial - una sucesión de golpes de teclas - no es necesariamente el resultado de un procesamiento serial, sino que puede explicarse también mediante un procesamiento paralelo. 
Se puede pensar que la mecanógrafa primero programa un golpe de letra, luego cl siguiente etc. Sin embargo, esto no es en realidad así: las manos y los dedos mientras se golpea una letra se anticipan para golpear la siguiente. Vamos a hacer un modelo de cómo la mente controla esta conducta.

Desde fuera del proceso de escribir a máquina viene la decisión de escribir una palabra. Este input activa un patrón de fuerzas de conexión correspondiente a dicha palabra. Este patrón activa, a su vez, los patrones o unidades correspondientes a las letras que componen la palabra. La unidad para la primera letra inhibe las activaciones de las unidades de las otras letras. La unidad para la segunda inhibe también la actividad de la unidad para la tercera letra etc. Ahora bien, como resultado de las múltiples interacciones de activaciones e inhibiciones entre las fucrzas de conexión de las diferentes unidades, la unidad para la primera letra resulta la más activada (no sufre ninguna inhibición), menos activada la unidad para la segunda letra, aún menos la unidad para la tercera letra.

Cada unidad para una letra influencia la mano y el dedo implicado en escribirla. Una vez que el dedo está en la distancia adecuada para golpcar, se pulsa la tecla. El acto de golpear inhibe la activación de la unidad para la primera letra; esto es, de la letra ya escrita. Lo cual permite que la letra segunda tenga su unidad o patrón el más activado et. De este modo la activación distribuída y paralela explica no sólo que se escriba secuencialmente, sino que hay una anticipación para escribir las letras siguientes.

Como podemos ver, según este modelo, el control motor ocurre como en el sistema nervioso. Todo el sistema se activa y la interacción múltiple de las fuerzas de activación e inhibición realiza el control motor. Naturalmente un modelo conexionista es algo más complicado que lo que hemos expuesto aquí, pero este ejemplo, resumido y esquemático, puede darnos una idea de lo que es un modelo conexionista y cómo se diferencia de los modelos convencionales de la psicología cognitiva.

\section{4. ¿Es el conexionismo un cambio de paradigma de la psicología?}

W. Schneider (1987) ha echado sobre sus hombros la difícil tarea de contestar a semejante pregunta. Su contestación tiene el valor, independientemente de su valor intrínseco, de ser la respuesta de un psicólogo que ha sido un miembro importante de la psicología cognitiva.

El planteamiento de Schneider es sencillo. Veamos cuáles son las caracteristicas que definen un cambio de paradigma y si esas características se dan con la aparición del conexionismo, entonces tendremos que admitir que hay un cambio de paradigma. Si esas características no se dan, entonces no habría fundamento para hablar de un cambio de paradigma. Así, pues, veamos cuáles son las características que señalan un cambio de paradigma.

Según Kuhn (1962), tal como recoge Schneider en su artículo, en su obra "La estructura de las revoluciones científicas" tienen que darse cuatro características para que podamos decir que se da un cambio de paradigma. En primer lugar, que el paradigma anterior se haga borroso y, en consecuencia, se pierdan sus reglas normales de investigación. En segundo lugar, que el viejo paradigma comienza a no hacer posible la esperanza de marchar hacia adelante en el conocimiento científico de esa ciencia 
específica y que, frente a esa situación de desesperanza, aparezca la esperanza de que es posible marchar hacia adelante con el cambio que se da en la ciencia. En tercer lugar, que la comunicación entre los científicos se haga difícil, porque el cambio de un paradigma a otro hace que la comunicación sea inevitablemente parcial. Finalmente y en cuarto lugar, que se de un solapamiento, aunque parcial, entre los problemas que se pueden resolver por el antiguo paradigma y por el nuevo.

Según Schneider (1987) con la llegada del conexionismo se cumplen esas cuatro características que señala Kuhn para definir un cambio de paradigma a otro hace que la comunicación sea inevitablemente parcial. Finalmente y en cuarto lugar, que se de un solapamiento, aunque parcial, entre los problemas que se pueden resolver por el antiguo paradigma y por el nuevo.

Scgún Schneider (1987) con la llegada del conexionismo se cumplen esas cuatro características que señala Kuhn para definir un cambio de paradigma. En consecuencia cree que el conexionismo representa un cambio significativo de paradigma en la psicología actual, un cambio tan profundo como fue el cambio del conductismo a la psicología del procesamiento de la información y que puede ser que sea el cambio más importante en la psicología que los psicólogos actuales van a presenciar a lo largo de sus vidas. Pero veamos cuál es la argumentación de Schneider.

En primer lugar, el paradigma de la psicología cognitiva se ha hecho borroso y se dan anomalias en sus reglas de investigación. Así durante los afios setenta se ha ido pasando de los modelos de cajas y sus diagramas de flujo a una variedad de representaciones de modelos, como los niveles de procesamiento, los esquemas, las redes semánticas y los sistemas de producción. En pocas palabras, que si nos preguntamos cuál es el modelo que usa la psicología cognitiva para describir los procesós cognitivos, no sabemos bien si son cajas o redes semánticas u otra cosa. El paradigma, pues, aparece borroso. Además se ha producido una relajación en las normas de investigación hasta el extremo que muchos psicólogos ya no investigan los procesos cognitivos en los seres humanos explícitamente, sino en programas de ordenador. Se da pues la circunstancia de que la psicología cognitiva presenta un paradigma borroso y anomalías en la investigación.

En cuanto a la segunda caracteristica de cambio de paradigma es evidente que mientras el entusiasmo por el conexionismo es tan fuerte que los autores recomiendan prudencia cautela, la actitud hacia el paradigma de la psicología cognitiva es ya en muchos algo que puede resumirse en las palabras de R. Luccio (1982) citadas anteriormente: "creo no equivocarme si considero más que probable que el cognitivismo, tal como lo hemos conocido, ha agotado, o está a punto de agotar, su función" (p. 255).

Por otra parte, el conexionismo está introduciendo nuevo vocabulario, nuevo tratamiento matemático e incluso nueva metodología que son ajenas a la ciencia cognitiva anterior, por lo que el entendimiento del conexionismo desde la psicología cognitiva se hace parcial y difícil.

Finalmente y por referimos a la cuarta de las características señaladas por Kuhn, en el momento actual de la psicología es innegable el solapamiento de conexionismo y cognitivismo en cuanto a muchos de los problemas que pretenden estudiar, como es el caso realmente llamativo del aprendizaje. ¿Tendremos que admitir, en consecuencia, que el conexionismo es un nuevo paradigma y que la psicología cognitiva tal como ha 
sido hasta ahora está a punto de morir? Esta es una cuestión a la que algunos autores, como hemos ya señalado, contestan afirmativamente, pero que han de ser los hechos y el tiempo los que en definitiva terminen por aclarar la cuestión.

Es por otra parte es fácil de asegurar una controversia entre cognitivistas y conexionistas, quizá como la hubo entre el conductismo y cl cognitivismo. Hoy por hoy el conexionismo aparece con grandes esperanzas, pero todavía tiene que resolver el problema, en mi opinión, de no convertirse en una pura teoría de los procesamientos. Como señala Schneider (1987) si deseamos tener un modelo de la cognición humana es crucial que generemos predicciones verificables empiricamente. El conexionismo, pues, necesita para afianzar su establecimiento en la psicología de un serio y extenso bagaje experimental.

\section{El impacto social del conexionismo}

Quizá no sería completo una visión de conjunto sobre el conexionismo, sino no exponemos, aunque sea en pocas palabras, la transcendencia social que en nuestros días está teniendo este nuevo modo de hacer psicología.

En nuestro país no sé si ésta es la primera exposición que se hace del conexionismo, por cierto breve y limitada. Sin embargo en los Estados Unidos en el presente año 1987 ya se han impartido seminarios sobre conexionismo en un centenar de universidades y las obras fundamentales sobre el conexionismo alcanzan un número de ventas insospechado. Por otra parte, en sólo cinco años el conexionismo ha pasado de no existir a ocupar un lugar destacado en los congresos de psicología y, como señala Shneider (1987), en la Sociedad de la Ciencia Cognitiva ocupa ya el treinta y uno por ciento de su programa.

Pero si en lo académico y básico el impacto del conexionismo es grande, lo es también en el campo de las investigaciones aplicadas tecnológicas. Es importante entender la computación biológica y la cognición, pero también es importante conseguir mejor tecnología y en relación con este punto quisiera citar textualemente unas palabras de Schneider: "Si estos sistemas de aprendizaje pueden realizar actividades perceptuales y de aprendizaje que nosotros corrientemente asociamos con los humanos, este movimiento conexionista causará una segunda revolución del computador que podría ser más significativa que la primera" (p. 78).

Nada de extraño que los organismos militares y civiles que conceden ayudas a la investigación se estén interesando enormemente por el moviminto conexionista e inviertan ya en nuestros días importantes cantidades en la investigación dentro de este campo. Hoy por hoy los modelos conexionistas que se construyen son, por decirlo así, abstractos, porque no conocemos bien el Hardware neurofisiológico, ni sabemos todavía realizarlo en silicona. Estas son metas esperanzadas de la nueva investigación.

\section{6. ¿Es el conexionismo una vuelta al conductismo?}

También a esta pregunta se ha intentado contestar ya. Ahora la respuesta ha estado a cargo de W. Bechtel (1985). En general, si hay motivos para hacerse semejante pregunta es por dos razones. Primero, porque el conexionismo utiliza modelos asociacionistas. Segundo, porque el conexionismo ha puesto especial énfasis en el 
aprendizaje. Esto es aducir sólo dos razones, pero son dos poderosas razones.

No puede negarse que el conexionismo es una vuelta a un cierto tipo de asociacionismo. Las unidades elementales de los modelos de procesamiento distribuído paralelo puede concebirse como aquellas ideas del empirismo y asociacionismo inglés que se unían en virtud de la experiencia. Si esto es así, tiene que ser igualmente válido afirmar que en este sentido el conexionismo se acerca a la tradición conductista, puesto que ésta sostiene una asociación de estímulos y respuestas, de tal manera que sus modelos explicativos son tambićn asociacionistas.

Naturalmente que el conexionismo no es una conexión entre estímulos y respuestas públicamente observables, ni aun siquiera podríamos decir es algo fundamental en su concepción la conexión entre inputs y outputs. Las conexiones ahora son entre unidades que son como neuronas y que no son públicamente observables. La dificultad, sin embargo, no está tanto en que las unidades sean públicamente observables o no, pues en el conductismo se admitió condicionamientos encubiertos, sino en que la red de asociaciones no es ahora entre sensaciones o conductas ni las asociaciones se rigen por la semejanza ni la contigüidad. Las unidades elementales del conexionismo no son directamente inputs sensoriales o outputs motores y la asociación de fuerzas de activación se produce por un gradiente descendente y no por las leyes clásicas de asociación.

Los modelos de procesamiento distribuído paralelo son modelos de procesamiento interno, lo que pretenden estudiar es la estructura de la mente, proporcionar una estructura en que la actividad mental pueda ocurrir. En este sentido los modelos de procesamiento distribuído paralelo tienen quizá mayor ligazón con los modelos cognitivos que con los modelos estímulo y respuesta del conductismo.

Por otra parte, hay otro hecho que une al conexionismo con el conductismo y este hecho es el énfasis que el conexionismo pone en el aprendizaje. Mientras la psicología cognitiva soslayaba el tema del aprendizaje ya que los mecanismos de la mente tendían a tomarse como innatos, el conexionismo estudia un sistema de conexiones capaz de modificar las ya existentes y apreder otras nuevas explicando así mejor cómo es la estructura de la mente que media la producción de la conducta futura.

Ahora bien, volver al aprendizaje parece ser de alguna manera volver al conductismo. Sin embargo, también hay profundas diferencias. En el conductismo lo que se estudiaban eran las leyes que caracterizan la conducta de aprendizaje, en el conexionismo lo que se estudia es el desarrollo de una estructura interna. La pregunta ahora es icómo se almacena la información en un sistema dinámico y de unidades elementales? ¿Cuáles son los procedimientos por los que el sistema es capaz de aprender? Todo esto no es contigüidad, pero tampoco es almacenaje de reglas explícitas. Aprendizaje es lo que el sistema hace ante una situación particular.

La conclusión de todo esto dista mucho de estar clara. Sin duda el conexionismo se preocupa por la mente y la cognición, cosa que el conductismo no hizo; trata de unidades intrínsecas y no de estímulos y respuestas extrínsecas. Pero no cabe duda que el conexionismo es asociacionista y fundamentalmente es una teoría del aprendizaje.

\section{Conclusión}

Hoy no se puede decir que la psicología cognitiva ha muerto y que lo vivo es el co- 
nexionismo. Tampoco se puede decir que el conexionismo es conductismo. Ambas afirmaciones son tan extremadas que son falsas. Sin embargo, sí es verdad que el conexionismo está mucho más cerca del conductismo que lo estuvo nunca la psicología cognitiva. La conclusión de Bechtel es que el conexionismo es una saludable síntesis de las dos tradiciones: la conductista y la cognitivista.

Ciertamente el conexionismo no es el cognitivismo y no va en contra de esta afirmación el que el conexionismo se haya creado y desarrollado por los psicólogos y científicos cognitivos, porque también la psicología cognitiva fue creada y desarrollada por los psicólogos conductistas. Pero, si embargo, mantiene cosas esenciales de la psicología cognitiva, como el interés por la mente y la concepción computacional. Ciertamente también el conexionismo no es el conductismo, aunque introduce muchas cosas que fueron esenciales en la tradición conductista.

La conclusión de Bechtel de que el conexionismo es una síntesis de las tradiciones conductista y cognitivista, quizá sea demasiado simple y quizá sea sólo una verdad parcial. Hay además otra verdad muy importante: la íntima relación del conexionismo con la neurofisiología. 


\section{REFERENCIAS}

Anderson, J. A. (1983) Cognitive and psychological computation whith neural models. IEEE Transaction on Systems, Man \& Cybernetics. Vol. SMC 13. 5. 799-815.

Bechtel, W. (1985) Contemporary connectionism: are the new parallel distributed processing models cogntive or asscciationist? Behaviorism. 13.1.53-60

Feldman, J. A. \& Ballard, D. H. (1982) Connectionist models and their propicrties. Cognitive Science. 6 205-254.

Hebb, D. O. (1949) The organization of behavior. New York. Wilcy.

Hinton, G. E. \& Anderson, J. A. (eds). (1981) Parallel models of associative memory. Hillsdale, NJ. Erlbaum.

Jackson, J. H. (1932) Selected writings of John Hughlings Jackson. Hodder and Stoughton.

Kuhn, T. S. (1962) The structure of scientific revolutions. Chicago. University of Chicago Press.

Lashley, K. S. (1950) In search of the engram Symp. Soc. Exp. Biol. 4. Cambrige. Cambridge University Press.

Luccio, R. (1982) Psicología cognitivista. En P. Legrenzi: Storia della psicologia. Bolonia. Il Mulino.

Luria, A. R. (1966) Human brain and psychological processes. New York. Harper and Row.

Miller, G. A., Galanter, E. Pribram, K. H. (1960) Plans and Structure of behavior. New York. Holt.

Pylyshyn, Z. W. (1984) Computation and cognition: Towards a foundation for cognitive science. Cambridge. MA. Bradford Books. MIT Press.

Rosenblatt, F.: (1962) Principles of neurodynamics. N. York. Spartan.

Rumelhart, D. E. \& Mc Clelland, J. L.: (1986) Parallel distributed Processing: explorations in the microstructure of cognition. $2 \mathrm{v}$ Cambridge MA. MIT Press.

Schneider, W.: (1987) Connectionism: it is a paradigm shift for psychology? Behavior Research Methods, Instruments \& Computers. 19. 2.73-83. 